hep-th/0605162

\title{
Opening Mirror Symmetry on the Quintic
}

\author{
Johannes Walcher \\ School of Natural Sciences, Institute for Advanced Study \\ Princeton, New Jersey, USA
}

\begin{abstract}
Aided by mirror symmetry, we determine the number of holomorphic disks ending on the real Lagrangian in the quintic threefold. The tension of the domainwall between the two vacua on the brane, which is the generating function for the open GromovWitten invariants, satisfies a certain extension of the Picard-Fuchs differential equation governing periods of the mirror quintic. We verify consistency of the monodromies under analytic continuation of the superpotential over the entire moduli space. We reproduce the first few instanton numbers by a localization computation directly in the A-model, and check Ooguri-Vafa integrality. This is the first exact result on open string mirror symmetry for a compact Calabi-Yau manifold.
\end{abstract}

May 2006 


\section{Introduction and Summary}

It has long been suspected that the enumerative results about holomorphic curves obtained by mirror symmetry [1] could be extended to open Riemann surfaces, provided appropriate boundary conditions are imposed. In the A-model, and at lowest order in the string coupling expansion, the counting of holomorphic disks ending on Lagrangian submanifolds is the central ingredient in the definition of Floer homology and the Fukaya category [2], which appears on one side of the homological mirror symmetry conjecture [3]. From the physics perspective, the chief interest is to determine the superpotential on the worldvolume of D-branes wrapping the Lagrangian, with many applications in studies of $\mathcal{N}=1$ compactifications of string theory.

Until now, the program of extending mirror symmetry to the open string sector has been successfully implemented only in a rather limited set of examples with special, toric, symmetries $[4,5]$. While certain general structures could be extracted from the results obtained [6-8], and of course much is known in lower-dimensional situations $[9,10]$, it has remained unclear whether and how these ideas could be implemented for more general, in particular compact, Calabi-Yau threefolds. This is precisely what we do in this paper.

The Calabi-Yau manifold $X$ we will consider is the most popular quintic in $\mathbb{C P}^{4}$, and our Lagrangian $L$ will be the most canonical real locus inside of it. This CalabiYau-Lagrangian pair has been contemplated many times in the literature, starting with [11]. First exact results were obtained in [12], where D-branes wrapping $L$ where identified with certain RS boundary states at the Gepner point [13] (see also [14] for a complementary derivation of this result). In $[15,16]$, the continuation of these

boundary states over the moduli space was analyzed using matrix factorizations $[17,18]$ in the mirror B-model Landau-Ginzburg description. In particular, it was explained in [16] that the singularity in the D-brane moduli space at the Gepner point could be interpreted as a degeneration of the Morse-Witten-Floer complex that computes Floer homology. Living in the A-model, the Floer differential differs from the classical Morse differential by corrections from holomorphic disks ending on $L[19,2]$, which suggested that one should be able to turn these results into a computation of the number of holomorphic disks as coefficients in the appropriate large-volume expansion. We will fulfill this promise in the present work, although following a slightly different route.

The central technical discovery is that the spacetime superpotential on the brane 


\begin{tabular}{|l|l|l|}
\hline$d$ & number of disks $n_{d}$ & number of spheres \\
\hline 1 & 30 & 2875 \\
3 & 1530 & 317206375 \\
5 & 1088250 & 229305888887625 \\
7 & 975996780 & 295091050570845659250 \\
9 & 1073087762700 & 503840510416985243645106250 \\
11 & 1329027103924410 & 1017913203569692432490203659468875 \\
13 & 1781966623841748930 & $229948856813626664832516010477226554 \ldots$ \\
15 & 2528247216911976589500 & $562465682466848327417948393837157975 \ldots$ \\
17 & 3742056692258356444651980 & $146020747145890338745688881159596996 \ldots$ \\
19 & 5723452081398475208950800270 & $397016669854518762338361058844977288 \ldots$ \\
\hline
\end{tabular}

Table 1: The number (integral invariants) of holomorphic disks in $X$ ending on $L$, of degree $d$ (only odd $d$ are shown, for reasons explained in the text), and, for comparison, the number of holomorphic spheres in $X$, according to [1].

worldvolume, which is the generating function capturing the open string instanton information [20-22], satisfies a differential equation which is a simple extension of the standard Picard-Fuchs differential equation whose solutions are the periods of the holomorphic three-form on the mirror of the quintic. The possible origin of such differential equations is discussed in special circumstances in $[7,8]$ (see also [23, 24]). But for a general brane configuration, or when the ambient Calabi-Yau is compact, the existence of this differential equation is, to the very least, surprising. Perhaps the most novel aspect of the equation that we introduce in this paper is that large complex structure is not a singular point of maximal unipotent monodromy. However, this has excellent reasons for being so, as we will explain below.

Equipped with the differential equation, it is straightforward to extract the open string instanton numbers, and we can check the integrality property conjectured in [21]. We do all this in section 2, and display, for amusement, the results in table 1.

It is then also of interest to study the analytic properties of the brane superpotential over the entire Calabi-Yau moduli space, and not just around large volume. Referring to section 3 for details, we would like to point out two salient features here. Firstly, the domainwall tension is invariant under monodromy around the conifold singularity in the moduli space. To appreciate the consistency of this result, one has to remember that the cycle that shrinks to zero volume at the conifold singularity in Kähler moduli 
space is a holomorphic cycle which can be wrapped by a B-brane, and it would be somewhat non-obvious why an A-brane would feel this singularity.

The second interesting feature is that the domainwall tension is not invariant under monodromy around the small-volume Gepner point. This is more surprising because, based on the worldsheet results of [16], one would have naively expected the domainwall tension to vanish at that point where the two vacua on the brane become degenerate. Instead, what happens is that the tension of the domainwall, when analytically continued from large volume, becomes asymptotically equal to a particular closed string period, which measures flux superpotentials. In other words, this domainwall only mediates a transition between different flux sectors, and this is still consistent with the degeneracy of the open string vacua. What it tells us, however, is that it could be much more delicate to understand our results from the worldsheet perspective, which is purportedly insensitive to the flux. It also indicates that it might be appropriate to include some of the flux data into the definition of Floer homology and the Fukaya category.

While this derivation of the superpotential and the instanton series can perfectly well stand alone, the confidence in the enumerative results of table 1 of course increases dramatically if at least some of those numbers can be verified mathematically directly in the A-model. We will do this in section 4.

The mathematical definition of open Gromov-Witten invariants in general still appears lacking [2], although several special cases have been treated in the literature. Studies of the local toric situation include [25-28]. Recently, Solomon [29,30] has performed a rigorous study of open Gromov-Witten invariants in the situation in which the Lagrangian providing the boundary conditions arises as the fixed point set of an anti-holomorphic involution. This covers the situation of our interest, so we can be confident that the numbers we are claiming are well-defined.

To go ahead with the direct computation of those open Gromov-Witten invariants, one can exploit the fact that, at least in our situation, any holomorphic mapping from the disk into $X$ with boundary on $L$ factors through a holomorphic sphere in $X$ meeting $L$ in a circle. In other words, we can relate the enumeration of holomorphic disks to the enumeration of holomorphic spheres which are invariant under the anti-holomorphic involution. For this problem, we have at our disposal the powerful graph combinatorial method introduced in [31]. This technique computes the Euler characteristic of a particular bundle on the moduli space $\mathcal{M}\left(\mathbb{C P}^{4}\right)$ of holomorphic curves in $\mathbb{C P}^{4}$ by using 
Atiyah-Bott localization with respect to the action of the torus $\left(S^{1}\right)^{5} \subset U(5)$ inside the symmetry group of $\mathbb{C P}^{4}$. The anti-holomorphic involution then acts in a natural way on this moduli space and the bundle over it, and one can identify the open GromovWitten invariant as the Euler characteristic of the resulting real bundle over the real locus in $\mathcal{M}\left(\mathbb{C P}^{4}\right)[32]$.

There are then two key points to appreciate in order to proceed. The first one is that while the anti-holomorphic involution breaks some of the symmetries of the ambient space, it still leaves an $\left(S^{1}\right)^{2} \subset O(5)$ unbroken. In particular, the fixed points on the real slice with respect to this torus coincide with the real fixed points of the torus in the complex case. The second point is that the Euler class of a real bundle is the squareroot of the Euler class of its complexification, where the sign is determined by the choice of orientation. With these two ingredients, it is straightforward to adapt the methods of [31] to develop a graphical calculus which computes the open GromovWitten invariants of our interest. We have checked that up to degree 7 , these numbers coincide with those obtained using mirror symmetry. The number (30) of holomorphic disks of degree 1 was first computed (without using localization) by Solomon [32,30]. We have also checked the number (1530) of holomorphic disks in degree 3 by taking a real slice of the localization computation of [33] on the space of curves (instead of the space of maps).

Besides the many possible applications and extensions of these results that spring to mind, we would like to mention that the numbers we get in this paper can also be viewed as providing lower bounds in real enumerative geometry in the sense of, see, e.g., $[34,35]$.

\section{The problem and its solution}

We consider in $\mathbb{C P}^{4}$ the Calabi-Yau hypersurface given as the vanishing locus of a polynomial of degree 5 in the homogeneous coordinates of $\mathbb{C P}^{4}$ :

$$
X=\left\{P\left(z_{1}, \ldots, z_{5}\right)=0\right\} \subset \mathbb{C P}^{4}
$$

The choice of $P$ determines the complex structure of $X$, and to define a $\sigma$-model with target space $X$, we need to pick a choice of complexified Kähler form $B+i J=t \omega$, where we denote by $\omega$ the integral generator of $H^{2}(X, \mathbb{Z})=\mathbb{Z}$, and $t$ is the Kähler parameter. 


\subsection{On the real quintic}

We want to identify in $X$ a particular Lagrangian submanifold as the fixed-point locus of an anti-holomorphic involution which acts on the ambient $\mathbb{C P}^{4}$ as complex conjugation on the homogeneous coordinates

$$
\left[z_{1}: z_{2}: \cdots: z_{5}\right] \mapsto\left[\bar{z}_{1}: \bar{z}_{2}: \cdots: \bar{z}_{5}\right]
$$

The complex structure on $X$ will be (anti-)invariant under this involution if the defining polynomial $P$ is real, in the sense that all its coefficients are real (up to a common phase). The fixed point locus, $L$, where $z_{i}=x_{i}$ is real is then given by the corresponding real equation $P\left(x_{1}, \ldots, x_{5}\right)=0$ inside of $\mathbb{R P}^{4} \subset \mathbb{C P}^{4}$. Straightforwardly, $L$ is a Lagrangian submanifold of $X$. In fact, $L$ is even special Lagrangian with respect to the holomorphic three-form on $X$.

Now while the topology of $X$ is well-known and independent of the complex structure, the real locus $L$ can have various topologies and singularities, with interesting transitions between them as $P$ is varied. We will not attempt to discuss all the possibilities here, but wish to comment on the consequences. To fix ideas, let us consider the Fermat quintic

$$
P=z_{1}^{5}+z_{2}^{5}+z_{3}^{5}+z_{4}^{5}+z_{5}^{5}
$$

Over the reals, $z_{i}=x_{i}$, we can solve for $x_{5}$ uniquely in terms of $x_{1}, \ldots x_{4}$, not all of which can be zero, lest $x_{5}$ will be zero too. This identifies $L$ with a copy of $\mathbb{R P}^{3}$. However, this identification depends on the fact that $z_{5}^{5}=a$ for real $a$ has only one real root, which will not be useful for a generic $P$.

There are at least two things that can happen to the real locus as we vary the complex structure. The first one is familiar from studies of stability conditions on Lagrangian submanifolds, and happens along a real codimension one locus in complex structure moduli space. When crossing such a wall of marginal stability, the special Lagrangian $L$ develops a singularity and reconnects on the other side, changing its topological type (but not its homology class). The second effect is a remnant of the standard conifold singularity in the complex structure moduli space. (It might seem that since the discriminant locus is complex codimension one, it would generically be missed by the half-dimensional real subspace. But this is untrue.) It was shown in [36] using a local model that when crossing such a conifold singularity, the homology class of the real locus always changes by the homology class of the vanishing cycle. 
The second phenomenon is known to happen on the quintic [37], for example when crossing the standard conifold locus $\psi=1$ along the one parameter family $P \rightarrow$ $P-5 \psi z_{1} z_{2} z_{3} z_{4} z_{5}$. Since the Lagrangian is connected at $\psi=0$, it implies that we must also be crossing a line of marginal stability somewhere between $\psi=0$ and $\psi=\infty$.

In this paper, we are studying $L$ in the A-model, and those aspects should be independent of the complex structure of $X$, and only depend on the Hamiltonian deformation class of $L$. Namely, we would expect to only depend on $L$ being Lagrangian, and not the special Lagrangian property. On the other hand, the available definitions of Floer homology for Lagrangians clearly depend on the underlying topology. (For instance, they depend on $b_{1}(L)$.) It is therefore not a priori clear why there should be a well-defined and invariant notion of Floer homology or of the "number of disks" ending on "the real locus $L$ " which is independent of the complex structure of $X$. One might worry slightly less about this in regard to the first phenomenon (marginal stability) because at least the homology class is preserved. In this paper, in any case, we will ignore this complication, and just pretend that $L \cong \mathbb{R P}^{3}$. The number of disks we will quote can then be understood as referring to "the generic quintic in a neighborhood of the Fermat point".

For the rest of the paper, we will be concerned with the dependence on the Kähler parameter, $t$, or its exponentiated version $q=e^{2 \pi i t}$. We begin in the large volume limit $q \rightarrow 0$.

\subsection{Vacuum structure at large volume}

Recall that to wrap an A-brane on $L$, we also need to specify a $U(1)$ bundle with a flat connection. Since $H_{1}(L ; \mathbb{Z})=\pi_{1}(L)=\mathbb{Z}_{2}$ we have two possible choices which are distinguished by a "discrete Wilson line", $W=\epsilon= \pm 1$. In fact, these two choices correspond to topologically distinct bundles on $\mathbb{R P}^{3}$, as measured by the first Chern class $c_{1} \in H^{2}(L ; \mathbb{Z})$. The latter is equal to $H_{1}(L ; \mathbb{Z})$ by Poincaré duality. On the

other hand, the K-theory of the quintic does not contain any torsion elements, and the two choices of flat connection can therefore not be distinguished by any topological charge [12].

As a consequence, when wrapping a D6-brane of type IIA string theory on $L$, the brane worldvolume will support an $\mathcal{N}=1$ gauge theory with two vacua corresponding to the two possible discrete Wilson lines, which are not distinguished by any conserved charge. We can then ask about the existence of a BPS domainwall that communicates 
between these two vacua.

To represent this domainwall in string theory, it is helpful to understand why the two bundles are topologically equivalent after inclusion in the quintic. Let us consider the situation with "non-trivial" Wilson line $\epsilon=-$ (we will see in a moment that this isn't really an invariant notion). The non-trivial first Chern class of the bundle on $L$ can be viewed as resulting from dissolving into the D6-brane a D4-brane wrapping the non-trivial one-cycle in $H_{1}(L ; \mathbb{Z})$. But since the quintic does not contain any non-trivial one-cycles, we can also contract it away to nothing.

Clearly, then, the BPS domainwall that mediates between the two choices of Wilson line on the D6-brane wrapping on $L$ is a D4-brane wrapping a holomorphic disk $D$ in $X$ with boundary on the non-trivial one-cycle in $L$ and extended along a $(2+1)$ dimensional subspace of Minkowski space. This D4-brane is a magnetic source on the D6-brane and hence changes the (discrete) magnetic flux on $L$. The topological classification of $D$ is as a non-trivial relative cohomology class in $H_{2}(X, L ; \mathbb{Z})$ with non-trivial image in $H_{1}(L ; \mathbb{Z})$.

It is not difficult to get a first approximation to the tension, $\mathcal{T}$, of this domainwall in the large volume limit (here and throughout the paper, we will refer to the tension as the holomorphic quantity whose absolute value gives the physical tension). Since $L$ is defined as the fixed point locus of an anti-holomorphic involution of $X$, any holomorphic disk ending on $L$ can be complex conjugated to a second holomorphic disk, and thereby completed to a holomorphic sphere. From the exact sequence

$$
H_{2}(X ; \mathbb{Z}) \rightarrow H_{2}(X, L ; \mathbb{Z}) \rightarrow H_{1}(L ; \mathbb{Z})
$$

we see that in fact also a brane wrapped on twice the generator of $H_{2}(X, L ; \mathbb{Z})$ will not change the vacuum on the brane, and hence be equivalent to a holomorphic sphere. The tension of that sphere being $t$ (the Kähler parameter), we infer $2 \mathcal{T} \sim t$.

To see that this argument was in fact quite incomplete, we need another fact about the relation between the cohomology of $X$ and that of $L$. Namely, when intersecting a hyperplane in $\mathbb{C P}^{4}$ with the Lagrangian $L$ (the hyperplane has to be represented by a complex linear equation in order to intersect $L$ transversely), we can see that the intersection locus is a non-trivial one-cycle in $L$. The Poincaré dual statement is that the integral generator of $H^{2}(X ; \mathbb{Z})$ restricts on $L$ to the non-trivial element of $H^{2}(L ; \mathbb{Z})$. Since the gauge invariant gauge field on the brane is $B-F$, this means that changing the flat $B$-field on $X$ by one unit is equivalent to exchanging the two flat gauge fields on the brane. 
A more elementary way to see this is to note that the path-integral contribution of a disk worldsheet wrapped on $D$ has a contribution $e^{2 \pi i t / 2}=q^{1 / 2}$ from its area and a contribution $\epsilon= \pm 1$ from its boundary, so changing $B \rightarrow B+1$ is equivalent to changing $\epsilon \rightarrow-\epsilon$. Taking $B \rightarrow B+2$ does nothing on the brane. In this sense, we can specify the Wilson line on the brane only after fixing the sign of $q^{1 / 2}$.

Now claiming that $\mathcal{T} \sim t / 2$ raises a puzzle because it is not invariant under $t \rightarrow t+2$. To resolve this, we have to note that the $\mathrm{D} 4$-brane wrapped on $D$ is a magnetic source not only for the gauge field on $L$, but also for the Ramond-Ramond 3-form field (we actually used this above to derive $2 \mathcal{T} \sim t$ ). The change of $\mathcal{T}$ under $t \rightarrow t+2$ is then explained by the non-invariance of RR flux under $B$-field monodromies.

So to make the formula for $\mathcal{T}$ more precise, and work out the spectrum of domainwalls, we have to include the RR flux quantum numbers in our labeling of the vacua. For the time being, 4-form flux, $N_{4}$, and 6-form flux, $N_{6}$, (around the unique four and 6-cycle of $X)$ will suffice, so our vacua are labeled as $\left(N_{4}, N_{6}, \epsilon\right)$.

We then require that a domainwall represented by a D4 wrapping an elementary disk $D$ connects $\epsilon-\epsilon$, and that by juxtaposing two such disks we obtain a sphere across which the only change is $N_{4} \rightarrow N_{4}+1$, that the B-field monodromy $B \rightarrow B+1$ changes $N_{6} \rightarrow N_{6}+N_{4}$, and also $\epsilon \rightarrow-\epsilon$, but is otherwise a symmetry of the spectrum. We also wish to keep 4- and 6-form flux integrally quantized to avoid concluding with fractional D0-branes.

It then turns out that, up to parity, there is only one consistent solution to these constraints. The change in 4-form flux across a D4-brane wrapped on $D$ is zero when $\epsilon=-$ on the left of the domainwall and it is equal to +1 when $\epsilon=+$ on the left, and, we have to let the $B$-field monodromy change the 4-form flux, in a way depending on $\epsilon$ :

$$
\begin{aligned}
\left(N_{4}, N_{6},-\right) & \rightarrow\left(N_{4}, N_{6}+N_{4},+\right) \\
\left(N_{4}, N_{6},+\right) & \rightarrow\left(N_{4}+1, N_{6}+N_{4},-\right)
\end{aligned}
$$

Let us denote the tension of a domainwall between vacuum $\left(N_{4}, N_{6}, \epsilon\right)$ on the left and vacuum $\left(N_{4}^{\prime}, N_{6}^{\prime}, \epsilon^{\prime}\right)$ on the right by $\mathcal{T}_{\left(N_{4}, N_{6}, \epsilon\right) \mid\left(N_{4}^{\prime}, N_{6}^{\prime}, \epsilon^{\prime}\right)}$. The above constraints are enough to determine all $\mathcal{T}$ 's as a function of $t$.

For example, let us consider the most basic $\mathcal{T}_{-} \equiv \mathcal{T}_{(0,0,-) \mid(0,0,+)}$ and $\mathcal{T}_{+} \equiv$ $\mathcal{T}_{(0,0,+) \mid(1,0,-)}$. Since $\mathcal{T}_{-}(t+1)=\mathcal{T}_{+}(t)$ and $\mathcal{T}_{+}+\mathcal{T}_{-}=t$, we conclude

$$
\mathcal{T}_{-}=\frac{t}{2}-\frac{1}{4} \quad \mathcal{T}_{+}=\frac{t}{2}+\frac{1}{4}
$$


Finally, we can write down the spacetime superpotential, which follows from (2.6) together with

$$
\mathcal{T}_{\left(N_{4}, N_{6}, \epsilon\right) \mid\left(N_{4}^{\prime}, N_{6}^{\prime}, \epsilon^{\prime}\right)}(t)=\mathcal{W}_{N_{4}^{\prime}, N_{6}^{\prime}, \epsilon^{\prime}}(t)-\mathcal{W}_{N_{4}, N_{6}, \epsilon}(t)
$$

We find

$$
\mathcal{W}_{N_{4}, N_{6},+}(t)=\frac{t^{2}}{4}+N_{4} t+N_{6} \quad \mathcal{W}_{N_{4}, N_{6},-}(t)=\frac{t^{2}}{4}-\frac{t}{2}+\frac{1}{4}+N_{4} t+N_{6}
$$

Of course, in this section, the discussion has been entirely classical and restricted to the large volume limit $t \rightarrow i \infty$. We now proceed to study the corrections $\mathcal{W}^{\text {quant. from }}$ worldsheet instantons.

\subsection{Worldsheet instanton corrections}

According to general philosophy $[22,21,20,4,38]$, the spacetime superpotential on the worldvolume of a particular supersymmetric brane wrapping a cycle in a Calabi-Yau manifold, $X$, when expressed in the A-model, and expanded in the appropriate variables, becomes the generating function counting worldsheet instanton corrections from holomorphic disks ending on the Lagrangian, L. Such a statement is in line with the role that holomorphic disks play in the definition of Fukaya's $A_{\infty}$ category [2], and the relationship between $A_{\infty}$ algebras and D-brane superpotentials [39,40].

More precisely, the spacetime superpotential can be identified with the topological disk partition function and is conjectured to admit an expansion of the general form

$$
\mathcal{W}(t, u)=F_{\text {disk }}(t, u)=\sum_{d, e} \tilde{n}_{d, e} q^{d} y^{e}=\sum_{d, e} \sum_{k \geq 1} \frac{n_{d, e}}{k^{2}} q^{k d} y^{k e}
$$

Here, the sum is over relative cohomology classes in $H_{2}(X, L), q=e^{2 \pi i t}$ is the (collection of) closed string Kähler parameters of $X$ and $y=e^{2 \pi i u}$ is the (collection of) exponentiated classical open string deformation parameters. The latter come from non-Hamiltonian deformations of the Lagrangian. They are $b_{1}(L)$ in number and are complexified by the Wilson line of the gauge field around the corresponding one-cycles of $L$. The final transformation in (2.9) is a resummation of multi-cover contributions and the central part of the conjecture is that the resulting expansion coefficients $n_{d, e}$ are integers [21] (whereas the $\tilde{n}_{d, e}$ are in general rational numbers). These integers have a spacetime interpretation as counting the "degeneracy of BPS domainwalls" in the class $(d, e)$. 
The existence and integrality of such an expansion has been checked in many examples involving local toric Calabi-Yau manifolds. Our goal in this paper is to make sense of and evaluate the formula (2.9) for the Calabi-Yau-Lagrangian pair $(X, L)=$ (quintic, real locus). At first sight, the fact that we only have a discrete open string modulus at our disposal is a deficiency because (2.9) makes explicit only rational cohomology. On second thought, however, it's a blessing.

For example, as we have discussed above, domainwalls arising from D4-branes wrapping holomorphic disks are sources for both the Ramond-Ramond field and the gauge field on the brane. But if the disk ends in a rational cycle of $L$, the gauge flux is non-zero as a differential form. This raises a puzzle because according to the standard worldsheet analysis, gauge fields on Lagrangian A-branes should be flat. From the spacetime perspective, this might well be repaired by a careful analysis of the couplings of the brane to the Ramond-Ramond fields. But it is clearly not obvious to see that from the TFT on the worldsheet. In the cases discussed in the literature (see $[4,5]$ and follow-up work), this problem is avoided because the Lagrangians considered there are non-compact and hence the flux can disperse to infinity.

A second advantage of having $H_{1}(L, \mathbb{Z})=\mathbb{Z}_{2}$ being torsion has to do with certain puzzlements [32] about the multi-cover formula as well as the integral "framing" ambiguity of open string amplitudes discovered in [5]. We do not understand either of those issues sufficiently well enough to usefully discuss here, but the consistency of our results indicates that both problems are absent for $H_{1}(L)=\mathbb{Z}_{2}$.

Finally, because our Lagrangian is compact, we can also discuss the classical contributions to the superpotential, as we have done in the previous subsection. The structure of these classical terms (which are absent from (2.9)) will help us to normalize the computation by imposing consistency of the monodromies around the various singular loci in the Kähler moduli space (see section 3).

So what is the possible structure of worldsheet instanton corrections to our formulas (2.6) for the domainwall tensions?

Clearly, the first non-trivial term will arise from worldsheet disks wrapped in the class $D$ generating $H_{2}(X, L ; \mathbb{Z})=\mathbb{Z}$, and will contribute at order $q^{1 / 2}$. Then there will be higher order terms. Let us call disks contributing at order $q^{d / 2}$ "of degree $d$ ". It is easy to see that the conditions $\mathcal{T}_{-}(t+1)=\mathcal{T}_{+}, \mathcal{T}_{+}+\mathcal{T}_{-}=t$ that we have used to derive (2.6) hold also after inclusion of non-perturbative worldsheet corrections. This is because $t$ is essentially defined to be the parameter measuring the tension of the 
domainwall wrapped on a degree 1 rational curve. The only form of the instanton expansion that is consistent with those constraints is that there are no contributions from even degree disks. This is in fact not unexpected, because disks of even degree have trivial boundary on the Lagrangian, and even though we can contemplate holomorphic disks of even degree ending on $L$, the triviality of their boundary makes it difficult to keep them there as we vary the complex structure of the quintic. In other words, we do not expect any invariant to exist for even degree.

So we expect a result of the form

$$
\mathcal{T}_{ \pm}=\frac{t}{4} \pm \frac{1}{2} \pm \text { const. } \sum_{d \text { odd }} \tilde{n}_{d} q^{d / 2}
$$

where the $\tilde{n}_{d}$ are certain rational numbers such that rewriting them as in (2.9),

$$
\tilde{n}_{d}=\sum_{k \mid d} \frac{n_{d / k}}{k^{2}}
$$

the $n_{d}$ turn out integer.

\subsection{Mirror Symmetry and open Picard-Fuchs equation}

The easiest way to get an expansion of the form (2.10) is to make use of mirror symmetry. What this means concretely is that we should first identify an object in the D-brane category which appears on the B-model side of the homological mirror symmetry conjecture, and which, via the equivalence of categories and up to auto-equivalences, corresponds to the object of the (derived) Fukaya category that is defined by $L$. We should then compute the appropriate superpotential/domainwall tension quantity as a function of the mirror parameter $\psi$ and reexpress it in terms of the flat coordinate $t$.

The Calabi-Yau mirror, $Y$, to the quintic is of course well-known. It is the resolution of a $\left(\mathbb{Z}_{5}\right)^{3}$ quotient of the one-parameter family of quintics $\sum z_{i}^{5}-5 \psi \prod z_{i}=0$ in $\mathbb{C P}^{4}$. Equivalently, we can consider a Landau-Ginzburg orbifold model with superpotential $W=\sum z_{i}^{5}-5 \psi \prod z_{i}$ and orbifold group $\left(\mathbb{Z}_{5}\right)^{4}$. The corresponding B-model category which is conjectured [41] to be equivalent to the derived category of $Y$ is the category of $\left(\mathbb{Z}_{5}\right)^{4}$ equivariant matrix factorizations of the superpotential $W$. (The corresponding equivalence was proven for the quintic itself by Orlov [42].)

And in fact, as we have mentioned in the introduction, the matrix factorization which is mirror to the Lagrangian $L$ is known explicitly (see $[15,16]$ for details). Given this identification of the matrix factorization and the equivalence with the derived 
category, it should be possible in principle to also describe explicitly a coherent sheaf on $Y$ corresponding to $L$. This would in fact be very interesting, because it would allow making use of some of the well-known machinery of holomorphic vector bundles that applies to problems of this type. In particular, there is an explicit formula for the superpotential, namely, the holomorphic Chern-Simons functional [11]

$$
\mathcal{W}^{B}=S_{\mathrm{hCS}}\left(A, A_{0}\right)=\int \Omega \wedge \operatorname{Tr}\left[A \wedge \bar{\partial}_{A_{0}} A+\frac{2}{3} A \wedge A \wedge A\right]
$$

No such expression is known in the matrix factorization formulation, and although there are formulas for TFT correlators $[43,44]$, they do not appear sufficient to determine the full superpotential. (See, however [45] for recent progress in making the $A_{\infty}$ constraints of [46] useful for this type of question.)

Leaving these explicit B-models for future investigations, we will instead obtain sufficient guidance from the non-compact examples of open mirror symmetry introduced in [4], and studied in depth in $[5,6,23,24,7,8]$.

The main simplification that occurs in these examples is that the B-model contains only D5-branes wrapped on curves in the Calabi-Yau. For such a brane configuration, the holomorphic Chern-Simons action (2.12) reduces to a "partial period" integral of the type

$$
\mathcal{W}\left(C, C_{*}\right)=\int_{\gamma} \Omega
$$

where $\gamma$ is a three-chain in $X$ with boundary $\partial \gamma=C-C_{*}$ equal to the difference of two possible positions of the D5-branes. (If $C$ and $C_{*}$ are holomorphic, (2.13) is literally the tension of the domainwall between the two vacua.) In the toric case, one can then further reduce the integral (2.13) to take place on a Riemann surface, so one has essentially a one-dimensional problem.

This structure was exploited in $[7,8]$ to show that the differential equations obtained in $[6,24]$ could be viewed as resulting from a certain variation of mixed Hodge structure on a certain relative cohomology. Explicitly, one retains the boundary terms arising in the derivation of the GKZ differential system and converts them into appropriate boundary variations. The upshot is that the open string mirror computations in the local toric case can be cast in a form very similar to the standard, closed string computations, involving Picard-Fuchs differential equations, maximal unipotent monodromy, mirror map, etc.. This is called $\mathcal{N}=1$ special geometry.

We do not know at present whether such considerations make sense for the general B-model situation. The case at hand, however, is sufficiently well constrained by our 
results so far that assuming the existence of a differential equation with properties as in $[7,8]$, there is essentially a unique candidate. This moreover turns out to produce excellent results.

The central idea of $\mathcal{N}=1$ special geometry is to extend the standard period vector by certain "partial periods" encoding information about the open string sector. We recall that in standard $(\mathcal{N}=2)$ special geometry, we have two periods for every closed string modulus, plus one or two extra ones related to the holomorphic three-form. In $\mathcal{N}=1$ special geometry, we gain one "partial period" for every classical open string modulus, plus one for every brane vacuum included in the background. Schematically,

$$
\Pi\left(t_{\text {closed }}, u_{\text {open }}\right)=\left(1, t_{\text {closed }}, \partial_{t} \mathcal{F}_{\text {closed }}, u_{\text {open }}, \mathcal{W}_{\text {brane }}, \ldots\right)^{T}
$$

where $\mathcal{F}_{\text {closed }}$ is the standard prepotential and the $u_{\text {open }}$ are the flat coordinates of the open string sector. The important point is that the period vector (2.14) satisfies a certain extension of the Picard-Fuchs differential equations. This differential system has all of the closed periods as solutions, plus extra ones related to $u_{\text {open }}$ and $\mathcal{W}_{\text {brane }}$. The latter gives the open string instanton expansion according to (2.9).

In the case that we have discussed in the previous subsections, we are not adding any classical open string modulus because $b_{1}\left(\mathbb{R P}^{3}\right)=0$, so the only modulus is the Kähler parameter $t$ of $X$, or equivalently, the mirror variable, $z=z(t)$. Moreover, according to (2.10), we need exactly one non-trivial domainwall tension as function of $t$ to encode the desired open string expansion. Let us call $\tau \sim q^{1 / 2}+\cdots$ the quantum part of the expansion (2.10). Since to leading order $z=q=e^{2 \pi i t}$, we will also have $\tau(z) \sim z^{1 / 2}+\cdots$ when expressed as a function of $z$.

Thus, we are simply seeking an ordinary linear differential equation in $z$, which, in addition to the four known periods of the mirror quintic, has exactly one additional linearly independent solution, $\tau$, with a squareroot behavior at $z=0$. The PicardFuchs equation governing periods of the mirror quintic being

$$
\mathcal{L} \varpi=\left[\theta^{4}-5 z(5 \theta+1)(5 \theta+2)(5 \theta+3)(5 \theta+4)\right] \varpi=0
$$

where $\theta=z \partial_{z}$, and $z=(5 \psi)^{-5}$, virtually the only possible extension that satisfies our constraints is the differential operator

$$
(2 \theta-1) \mathcal{L}=(2 \theta-1) \theta^{4}-5 z(2 \theta+1)(5 \theta+1)(5 \theta+2)(5 \theta+3)(5 \theta+4)
$$

We will now analyze this differential equation and show that it satisfies all the other desirable properties as well. 


\subsection{The instanton sum}

We follow conventions of [1]. The differential equation $\mathcal{L} \varpi=0$ has one distinguished solution, called the fundamental period, which has a power series expansion around the large complex structure point $z=0$,

$$
-w^{2}(z) \equiv \varpi_{0}(z)=\sum_{m=0}^{\infty} \frac{(5 m) !}{(m !)^{5}} z^{m}
$$

All other solutions contain logarithms as $z \rightarrow 0$, large complex structure being a point of maximal unipotent monodromy. The period with a single logarithm, $w^{1}(z)$, has the information about the mirror map via $t=w^{1} / w^{2}, q \equiv e^{2 \pi i t}$.

$$
-2 \pi i w^{1}(z)=\varpi_{0}(z) \log z+5 \sum_{m=1}^{\infty} \frac{5 m) !}{(m !)^{5}} z^{m}[\Psi(1+5 m)-\Psi(1+m)]
$$

Under large complex structure monodromy, $z \rightarrow e^{2 \pi i} z, w^{1} \rightarrow w^{1}+w^{2}$ and $t \rightarrow t+1$.

There are then two further solutions of (2.15), both of which contain the closed string instanton information, in slightly different forms. Specifically, the solution of (2.15) called $\mathcal{F}_{1}$ in [1] is characterized by the boundary conditions

$$
(2 \pi i)^{2} \mathcal{F}_{1}=-5 \cdot(2 \pi i) w^{1}(z) \log z+\frac{5}{2} w^{2}(z)(\log z)^{2}-\frac{21}{2} \cdot(2 \pi i)^{2} w^{1}(z)+\mathcal{O}(z)
$$

It transform under large complex structure monodromy as $\mathcal{F}_{1} \rightarrow \mathcal{F}_{1}-5 w^{1}-8 w^{2}$. Finally, the solution called $\mathcal{F}_{2}$ in [1] is characterized by $\mathcal{F}_{2} \rightarrow \mathcal{F}_{2}-\mathcal{F}_{1}-3 w^{1}+5 w^{2}$ as $t \rightarrow t+1$.

These periods $\left(\mathcal{F}_{1}, \mathcal{F}_{2}, w^{1}, w^{2}\right)$ can be interpreted as the quantum corrected masses of D4, D6, D2 and D0-brane on the quintic, respectively [12]. They therefore also give the tension of domainwalls mediating between various flux sectors, including the corrections from worldsheet instantons. For example, in the proper Kähler normalization $w^{2}=1$, one obtains after inverting (2.18) and expanding in $q=e^{2 \pi i t}$,

$$
\frac{\mathcal{F}_{1}}{w^{2}}=-\frac{5}{2} t^{2}-\frac{21}{2} t+\frac{1}{4 \pi^{2}}\left[2875 q+\frac{4876875}{4} q^{2}+\cdots\right]
$$

The polynomial in $t$ is the classical tension from the geometric volume of the cycles

and the power series in $q$ gives the quantum corrections. The rational coefficient $\tilde{N}_{d}$ of $q^{d}$ in this expansion gives the contribution from holomorphic spheres of degree $d$. They satisfy the property that when reexpressed in terms of $N_{d}$ via

$$
\tilde{N}_{d}=\sum_{k \mid d} \frac{d N_{d / k}}{k^{3}}
$$


the $N_{d}$ are integers. Note that we have here slightly unconventionally expanded the first derivative of the prepotential instead of the prepotential itself or the Yukawa coupling as in [1]. Since periods and brane superpotentials are on equal footing in $\mathcal{N}=1$ special geometry, this will make the comparison with the open string version (2.25) more natural.

Turning now to the equation (2.16), it has, by construction, exactly one additional solution, which we normalize to $\tau(z)=z^{1 / 2}+\cdots$. We find,

$$
\tau(z)=\frac{\Gamma(3 / 2)^{5}}{\Gamma(7 / 2)} \sum_{m=0}^{\infty} \frac{\Gamma(5 m+7 / 2)}{\Gamma(m+3 / 2)^{5}} z^{m+1 / 2}
$$

In the next section, we will determine from monodromy calculations on the Kähler moduli space that $\tau$ enters the domainwall tension in the normalization

$$
\mathcal{T}_{ \pm}(t)=\frac{w^{1}}{2} \pm \frac{w^{2}}{4} \pm \frac{15}{\pi^{2}} \tau(z)
$$

This then has exactly the expected form (2.10). Consulting (2.6) and its relation with (2.8), we then conclude that the contribution of worldsheet disk instantons to the spacetime superpotential is

$$
\mathcal{W}^{\text {quant. }}=\frac{30}{4 \pi^{2}} \tau(z)
$$

Dividing by $w^{2}$ to go to the canonical normalization of the holomorphic three-form, multiplying by $4 \pi^{2}$ as in (2.20), inverting the mirror map, and doing the expansion, we obtain the open string instanton sum

$$
\hat{\tau}(q)=30 \frac{\tau(z(q))}{\varpi_{0}(z(q))}=30 q^{1 / 2}+\frac{4600}{3} q^{3 / 2}+\frac{5441256}{5} q^{5 / 2}+\cdots
$$

We can then plug in to $\hat{\tau}(q)$ the Ooguri-Vafa multi-cover formula (2.9)

$$
\hat{\tau}(q)=\sum_{\substack{d \text { odd } \\ k \text { odd }}} \frac{n_{d}}{k^{2}} q^{d k / 2}=\sum_{d \text { odd }} n_{d} \frac{q^{d / 2}}{4} \Phi\left(q^{d}, 2,1 / 2\right)
$$

where $\Phi$ is the Lerch Transcendent. For reasons explained in a previous subsection, we only consider disks of odd degree and their odd multi-covers. The first few $n_{d}$ are indeed integer and displayed in table 1 in the introduction.

It should be stressed that we have strictly speaking not shown that the constant normalization factor in (2.10) is equal to $\frac{1}{2 \pi^{2}}$ as claimed. It is, however, the most natural choice and consistent with everything else we know. It would be interesting to derive this value more directly. 


\section{Analytic continuation of the superpotential}

The purpose of this section is to analytically continue our result for the superpotential/domainwall tension over the entire quantum Kähler moduli space of the quintic, much as was done for the closed string periods in [1]. This will not only help us to fix the normalization factor anticipated in (2.23), but is interesting in its own right as it can shed light on intrinsically stringy aspects of D-brane physics that have hitherto been inaccessible. We will indeed find that the analytic properties of the $\mathcal{T}_{ \pm}$are rather interesting.

Recall that the Kähler moduli space of the quintic has three special points: large volume point $z \rightarrow 0$ that we have already discussed in depth, the conifold singularity $z=5^{-5}$ at which the period $\mathcal{F}_{2}$ vanishes, and the so-called Gepner or Landau-Ginzburg point, $z \rightarrow \infty$, which is not a singularity of the CFT, but exhibits a $\mathbb{Z}_{5}$ orbifold monodromy. We wish to understand the analytic behavior of $\mathcal{W}$, or equivalently $\mathcal{T}$, around each of these points. We shall work with the ansatz

$$
\mathcal{T}_{ \pm}(z)=\frac{w^{1}(z)}{2} \pm \frac{w^{2}(z)}{4} \pm a \tau(z)
$$

and determine the coefficient $a$ from consistency requirements.

The standard tool to do the analytic continuation of solutions of a hypergeometric differential equation of the type (2.16) is the Barnes integral representation. For $\tau$, this representation takes the form

$$
\tau(z)=\frac{\pi^{2}}{60} \frac{1}{2 \pi i} \int_{C} \frac{\Gamma(-s+1 / 2) \Gamma(5 s+1) \Gamma(s+1 / 2)}{\Gamma(s+1)^{5}} e^{i \pi(s-1 / 2)} z^{s}
$$

where the integration contour is straight up the imaginary axis. For $|z|<5^{-5}$, we close the contour on the positive real axis and recover (2.22). For $|z|>5^{-5}$, we instead close the contour on the negative real axis, and obtain the expansion

$$
\begin{aligned}
& \tau(z)=\tau_{1}(z)+\tau_{2}(z)=\frac{\pi^{2}}{60}\left[\sum_{m=0}^{\infty} \frac{-\Gamma(-5 m-3 / 2)}{\Gamma(-m+1 / 2)^{5}} z^{-m-1 / 2}\right. \\
&\left.+\sum_{m=1}^{\infty} \frac{-\Gamma(m / 5) e^{4 \pi i m / 5}}{5 \Gamma(m) \Gamma(1-m / 5)^{4}} z^{-m / 5} e^{-i \pi / 2} \frac{\sin \pi m / 5}{\cos \pi m / 5}\right]
\end{aligned}
$$

The first term, $\tau_{1}(z)$, is simply the unique solution of (2.16) with a squareroot behavior around $z=\infty$, and changes sign as we circle around $z^{1 / 5} \rightarrow e^{-2 \pi i / 5} z^{1 / 5}$. The second sum in (3.3) is easily verified to be a solution of the ordinary Picard-Fuchs equation, 
and hence a closed string period. To determine which one, we can compare it with the canonical $\mathbb{Z}_{5}$ symmetric basis of solutions of (2.15) around the Gepner point [1], $(j=0, \ldots, 4)$

$$
\varpi_{j}(z)=\sum_{m=1}^{\infty} \frac{-\Gamma(m / 5) e^{4 \pi i m / 5}}{5 \Gamma(n) \Gamma(1-m / 5)^{4}} z^{-m / 5} e^{2 \pi i j m / 5}
$$

Indeed, the identity

$$
\frac{\sin \pi m / 5}{\cos \pi m / 5}=2 \sin 2 \pi m / 5-2 \sin 4 \pi m / 5
$$

shows that

$$
\tau_{2}(z)=\frac{\pi^{2}}{60}\left[\varpi_{0}+2 \varpi_{4}+2 \varpi_{2}\right]
$$

According to the results of [1], the small volume period vector $\varpi=\left(\varpi_{2}, \varpi_{1}, \varpi_{0}, \varpi_{4}\right)^{T}$ is related to the large volume basis $\amalg=\left(\mathcal{F}_{1}, \mathcal{F}_{2}, w^{1}, w^{2}\right)^{T}$ via $\amalg=M \varpi$ with

$$
M=\left(\begin{array}{cccc}
\frac{3}{5} & \frac{1}{5} & -\frac{21}{5} & -\frac{8}{5} \\
0 & -1 & 1 & 0 \\
-\frac{1}{5} & -\frac{2}{5} & \frac{2}{5} & \frac{1}{5} \\
0 & 0 & -1 & 0
\end{array}\right)
$$

This allows us to express $\tau_{2}(z)$ in the integral basis,

$$
\tau_{2}(z)=\frac{\pi^{2}}{60}\left[-4 \mathcal{F}_{1}+8 \mathcal{F}_{2}-11 w^{1}+15 w^{2}\right]
$$

Moreover, by using the known monodromy matrices around the Gepner point, we find that as $z^{-1 / 5} \rightarrow e^{2 \pi i / 5} z^{-1 / 5}$,

$$
w^{1} \rightarrow-\mathcal{F}_{2}+w^{1}-w^{2}, \quad w^{2} \rightarrow \mathcal{F}_{2}+w^{2}, \quad \tau \rightarrow-\tau+\frac{\pi^{2}}{60} \mathcal{F}_{2}
$$

Thus we see that were it not for the quantum corrections of the domainwall tension in (3.1), the Gepner monodromy would take $\frac{w^{1}}{2}+\frac{w^{2}}{4}$ to $\frac{w^{1}}{2}-\frac{w^{2}}{4}-\frac{\mathcal{F}_{2}}{4}$, and would not induce a symmetry of the domainwall spectrum as it should. Moreover, we see that the lucky number that makes the Gepner monodromy integral is indeed $a=\frac{15}{\pi^{2}}$. (Strictly speaking, this is only the minimal possibility, a natural choice.) With this value, the Gepner monodromy acts as

$$
A: \quad \mathcal{T}_{+} \rightarrow \mathcal{T}_{-}, \quad \mathcal{T}_{-} \rightarrow \mathcal{T}_{+}-w^{2}-\mathcal{F}_{2}
$$


on the open string periods. Since as discussed in section 2, the large volume monodromy acts by $T_{\infty}: \mathcal{T}_{-} \rightarrow \mathcal{T}_{+}, \mathcal{T}_{+} \rightarrow \mathcal{T}_{-}+w^{2}$, we find by combining the two that the conifold monodromy about $z=5^{-5}, T=T_{\infty}^{-1} \circ A^{-1}$ acts trivially on both $\mathcal{T}_{+}$and $\mathcal{T}_{-}$.

Let us verify this last assertion explicitly, in order to check that everything is consistent. A straightforward way to compute this monodromy is to compare the divergence of the large volume expansions (2.17) and (2.22) as $z$ approaches the singularity $z \rightarrow z_{*}=5^{-5}$. We know from [1] that at the conifold, $\mathcal{F}_{2}$ vanishes as $\mathcal{F}_{2} \sim \alpha_{1}\left(z-z_{*}\right)+\alpha_{2}\left(z-z_{*}\right)^{2}+\cdots$ and $\varpi_{0}$ behaves as $\varpi_{0} \sim \frac{1}{2 \pi i} \mathcal{F}_{2} \log \left(z-z_{*}\right)+$ regular. To determine the coefficient $b$ in $\tau \sim \frac{b}{2 \pi i} \mathcal{F}_{2} \log \left(z-z_{*}\right)+$ regular, we compare the second derivatives of $\varpi_{0}$ and $\tau$ as $z \rightarrow z_{*}$. Using Stirling's formula, we find

$$
\varpi_{0}^{\prime \prime} \sim \sum_{m}\left(5^{5} z\right)^{m}\left[\frac{5^{10} \sqrt{5}}{4 \pi^{2}}-\frac{7 \cdot 5^{9} \sqrt{5}}{4 \pi^{2}} \frac{1}{m}+\cdots\right]
$$

which determines $\alpha_{1}, \alpha_{2}$. Doing the same for $\tau$ delivers

$$
\tau^{\prime \prime} \sim \sum_{m}\left(5^{5} z\right)^{m+1 / 2}\left[\frac{5^{10} \sqrt{5}}{4 \pi^{2}}-\frac{7 \cdot 5^{9} \sqrt{5}}{4 \pi^{2}} \frac{1}{m}+\cdots\right]
$$

This implies $b=1$.

Thus, we find that the conifold monodromy takes $\tau \rightarrow \tau+\frac{\pi^{2}}{60} \mathcal{F}_{2}$, and since $w^{2} \rightarrow$ $w^{2}-\mathcal{F}_{2}, \mathcal{T}_{ \pm}$are invariant when we set $a=\frac{15}{\pi^{2}}$.

It is also worth pointing out that for $a=\frac{15}{\pi^{2}}$, the leading behavior of $\mathcal{T}_{ \pm}$as $z \rightarrow$ $\infty$ is the same as that of an integral closed string period. This follows from (3.3) in conjunction with (3.8). As was mentioned in the introduction, this is a further consistency check on our results. It was shown in $[15,16]$ that the two open string vacua associated with the choice of discrete Wilson line (see subsection 2.2) could be identified with certain matrix factorization in the Landau-Ginzburg B-model. At the Gepner point, $z \rightarrow \infty$, the open string spectrum on the brane develops an extra massless state with a cubic superpotential. (This coalescence of open string vacua was first proposed in [12].) There should therefore be a domainwall between the two vacua that becomes tensionless as $z \rightarrow \infty$. Our result is then that while such a domainwall can indeed exist, it is not the most naive one obtained by wrapping a D4-brane on the primitive disk, but has to be combined with the appropriate integral period from (3.8).

To conclude this section, we summarize the results for the action of the monodromies around Gepner point, conifold point, and large volume point on the extended period vector (we now use $\mathcal{T}_{-}=-\mathcal{T}_{+}+w^{1}$ )

$$
\amalg=\left(\mathcal{T}_{+}, \mathcal{F}_{1}, \mathcal{F}_{2}, w^{1}, w^{2}\right)^{T}
$$


We have:

$$
\begin{gathered}
A \\
\left(\begin{array}{ccccc}
-1 & 0 & 0 & 1 & 0 \\
0 & 1 & 3 & 5 & 3 \\
0 & 1 & -4 & 8 & -5 \\
0 & 0 & -1 & 1 & -1 \\
0 & 0 & 1 & 0 & 1
\end{array}\right)\left(\begin{array}{ccccc}
1 & 0 & 0 & 0 & 0 \\
0 & 1 & 0 & 0 & 0 \\
0 & 0 & 1 & 0 & 0 \\
0 & 0 & 0 & 1 & 0 \\
0 & 0 & -1 & 0 & 1
\end{array}\right)\left(\begin{array}{ccccc}
-1 & 0 & 0 & 1 & 1 \\
0 & 1 & 0 & -5 & -8 \\
0 & -1 & 1 & -3 & 5 \\
0 & 0 & 0 & 1 & 1 \\
0 & 0 & 0 & 0 & 1
\end{array}\right)
\end{gathered}
$$

These matrices satisfy $A \cdot T \cdot T_{\infty}=1$ and $A^{10}=1$, but $A^{5} \neq 1$. Thus we find that the combined open-closed moduli space is a double cover of the quantum Kähler moduli space of the quintic, branched at $z=0$ and $z=\infty$.

\section{Localization in the A-model}

In this section we shall show how to check the enumerative predictions that we have obtained using mirror symmetry. We have outlined the main strategy in the introduction, so we will attempt to be brief. Details can be filled in from [31] and [19], Chapter 27.

Consider the moduli space $\mathcal{M}_{d} \equiv \overline{\mathcal{M}}_{0,0}\left(\mathbb{C P}^{4}, d\right)$ of genus zero stable maps to $\mathbb{C P}^{4}$ in degree $d$. For each point $f: \Sigma \rightarrow \mathbb{C P}^{4}$ in $\mathcal{M}$, we can pullback from $\mathbb{C P}^{4}$ the bundle $\mathcal{O}(5)$ of quintic polynomials. The global sections of that bundle $\mathcal{O}(5 d)$ over $\Sigma$ then fit together to a vector bundle $\mathcal{E}_{d}$ as we vary $f$ over $\mathcal{M}_{d}$. Any particular quintic polynomial $P\left(z_{1}, \ldots, z_{5}\right)$ in the homogeneous coordinates of $\mathbb{C P}^{4}$ gives a section of $\mathcal{O}(5)$. The resulting section of $\mathcal{E}_{d}$ vanishes at precisely those genus zero maps into $\mathbb{C P}^{4}$ which happen to be contained in the quintic given by $P$. This identifies the number of genus zero, degree $d$ maps to the quintic as the Euler class of $\mathcal{E}_{d}$ :

$$
\tilde{N}_{d}=\int_{\mathcal{M}_{d}} c_{5 d+1}\left(\mathcal{E}_{d}\right)
$$

It was shown in [31] that this Euler class can be very efficiently computed using AtiyahBott localization. The entire structure described above carries an $\left(S^{1}\right)^{5}$ action inherited from the standard $U(5)$ action on $\mathbb{C P}^{4}$. On the homogeneous coordinates, this torus acts as

$$
\mathbb{T}^{5}=\left(S^{1}\right)^{5} \ni\left(\rho_{1}, \ldots, \rho_{5}\right):\left[z_{1}: \cdots: z_{5}\right] \mapsto\left[\rho_{1} z_{1}: \cdots: \rho_{5} z_{5}\right]
$$

(This action can be complexified, of course, but we really only need the real torus.) On $\mathbb{C P}^{4}$, there are exactly five fixed points, $p_{i}$, of this torus action, defined by $z_{j}=0, j \neq i$. 
The fixed point loci on $\mathcal{M}_{d}$ can be associated combinatorially with certain decorated tree graphs, $\Gamma$. The vertices of these graphs (which can have arbitrary valence, val $(v)$ ) correspond to (genus 0) contracted components of the source $\Sigma$. They are labeled by one of the fixed points $p_{i}$ which tells where the component maps. The edges of the graph correspond to non-contracted rational components of $\Sigma$ mapping onto the coordinate line joining $p_{i}$ to $p_{j}$. They are labeled by a positive integer $d$ describing the degree of that map. The constraints on this decoration are that $p_{v} \neq p_{v^{\prime}}$ for adjacent vertices $v, v^{\prime}$ and that the sum of degrees on the edges be equal to the total degree under consideration.

In general, the fixed loci are not isolated points, but consist of certain moduli spaces $\overline{\mathcal{M}}_{\Gamma}$ arising from the contracted components at the vertices (of valence $\geq 3$ ). One can then compute the ( $\mathbb{T}^{5}$-equivariant) Euler class of the normal bundle of $\overline{\mathcal{M}}_{\Gamma}$ inside of $\mathcal{M}_{d}$, as well as the Euler class of $\mathcal{E}_{d}$ at the fixed points. The integrals over the $\overline{\mathcal{M}}_{\Gamma}$ can be done, and what results is a very explicit formula for $\tilde{N}_{d}$ given by a sum over graphs and labellings, divided by the appropriate symmetry factor.

We wish to accomplish something similar for the holomorphic maps of disks to the quintic with boundary on the real locus.

As we have indicated before, any disk with boundary on the real locus can be completed to a sphere, and the two halves of that sphere contribute in the same relative homology class. Conversely, any sphere of odd degree is cut in two by the real locus in a non-trivial one-cycle. ${ }^{1}$ Therefore, the number of disks of odd degree $d$ is equal to twice the number of spheres of degree $d$ which are invariant under complex conjugation of source and target. On the real locus $\mathcal{M}_{d}^{\mathbb{R}} \subset \mathcal{M}_{d}$, complex conjugation defines a real structure on the bundle of quintics $\mathcal{E}_{d}$ (and, of course, on the tangent bundle). Since we are interested in maps into a real quintic, we can identify the open Gromov-Witten invariant as [32]

$$
\tilde{n}_{d}=2 \int_{\mathcal{M}_{d}^{\mathbb{R}}} \mathbf{e}\left(\mathcal{E}_{d}^{\mathbb{R}}\right)
$$

In trying to apply localization to this problem, one is naively troubled by the fact that the torus action (4.2) does not commute with the standard complex conjugation (2.2). However, it is easy to realize that there is another real subtorus of $U(5)$ which does. This torus is two-dimensional and is the Cartan torus of $O(5) \subset U(5)$. It is the natural

\footnotetext{
${ }^{1}$ This is not true for even degrees: There can be real spheres of even degree without real points. In the real problem, they give rise to maps from the crosscap to the quintic. In other words, they will play a role in orientifolds. I am grateful to Jake Solomon for extensive discussions on these issues.
} 
four-dimensional analogue of the $S^{1}$ action used in [25]. An equivalent way to describe this is to choose the alternative complex conjugation

$$
\sigma: \quad\left[z_{1}: z_{2}: z_{3}: z_{4}: z_{5}\right] \mapsto\left[\bar{z}_{2}: \bar{z}_{1}: \bar{z}_{4}: \bar{z}_{3}: \bar{z}_{5}\right]
$$

which commutes with the subtorus $\mathbb{T}^{2}$ of (4.2) defined by $\rho_{2}=\rho_{1}^{-1}, \rho_{4}=\rho_{3}^{-1}, \rho_{5}=1$. The nifty thing about this torus is that its fixed points on $\mathbb{C P}^{4}$ are identical to those of (4.2). Moreover, it is not hard to see that the fixed points of $\mathbb{T}^{2}$ acting on $\mathcal{M}_{d}^{\mathbb{R}}$ are simply those fixed points of $\mathbb{T}^{5}$ acting on $\mathcal{M}_{d}$ which are invariant under $\sigma$.

From this discussion, we see that our task is to take a real section of Kontsevich's calculation [31] with respect to the complex conjugation $\sigma$. A moment's thought shows why this is feasible: Any $\sigma$-invariant decorated graph of odd total degree contains the real locus of $\Sigma$ at the middle of an edge. In other words, the contracted components of $\Sigma$ are away from the real locus. The upshot is that the integrals over the fixed loci are identical to those before.

To understand the Euler class of the normal bundle and of the bundle of real quintics, we are helped by the following elementary fact: If $V$ is any real vector bundle, then the square of its Euler class is the Euler class of its complexification,

$$
\mathbf{e}(V)=\sqrt{\mathbf{e}(V \otimes \mathbb{C})}
$$

For bundles of high enough rank, this formula of course only makes sense for the universal bundle, or in equivariant cohomology. The sign of the squareroot in (4.5) is determined by the choice of orientation on $V$ (which does not affect the canonical orientation of $V \otimes \mathbb{C}$ ). In our situation, $\mathcal{E}_{d}^{\mathbb{R}} \otimes \mathbb{C}=\left.\mathcal{E}_{d}\right|_{\mathcal{M}_{d}^{\mathbb{R}}}$ and since we already know $\mathbf{e}\left(\mathcal{E}_{d}\right)$, we are done.

Our graphical calculus is then very much as in [31]. A moduli space of $\mathbb{T}^{2}$-invariant disks corresponds to a tree graph $\Gamma$ with vertices mapping to fixed points $p_{\mu(v)}$ (with $\mu(v) \in\{1, \ldots, 5\})$ and edges mapping to coordinate lines joining $p_{i}$ to $p_{j}$. There is one special vertex, call it the first one, on which ends an extra half-edge with odd degree, call it $d_{0}$. This restriction is to ensure that the total degree

$$
d=d_{0}+2 \sum_{\text {edges }} d(e)
$$

can be odd. Another condition is that the special vertex cannot map to $p_{5}$. This arises from the fact that when we reconstruct a $\sigma$-invariant sphere by reflecting our graph on the half-edge, the first vertex will be adjacent to its image, and $\sigma\left(p_{5}\right)=p_{5}$. 
In taking a squareroot of the formulas in [31], we have to fix the signs. In principle, this could be done by a careful analysis such as advertised in $[29,30]$. In practice, the condition that the answer be independent of the torus weights is enough to determine the sign. Explicitly, we have

$$
\begin{gathered}
\int_{\overline{\mathcal{M}}_{\Gamma}} \frac{\mathbf{e}\left(\mathcal{E}_{d}^{\mathbb{R}}\right)}{\mathbf{e}\left(N_{\Gamma}^{\mathbb{R}}\right)}=\prod_{\text {edges }} \frac{\prod_{a=0}^{5 d} \frac{a \lambda_{i}+(5 d-a) \lambda_{j}}{d}}{(-1)^{d} \frac{(d !)^{2}}{d^{2 d}}\left(\lambda_{i}-\lambda_{j}\right)^{2 d} \prod_{\substack{k \neq i, j \\
a=0}}^{d}\left(\frac{a}{d} \lambda_{i}+\frac{d-a}{d} \lambda_{j}-\lambda_{k}\right)} \\
\cdot \prod_{\operatorname{vertices}} \frac{1}{\left(5 \lambda_{v}\right)^{\operatorname{val}(v)-1}} \prod_{j \neq v}\left(\lambda_{v}-\lambda_{j}\right)^{\operatorname{val}(v)-1} \cdot\left(\prod_{\text {flags }} \frac{d}{\lambda_{v}-\lambda_{j}}\right)\left(\sum_{\text {flags }} \frac{d}{\lambda_{v}-\lambda_{j}}\right)^{\operatorname{val}(v)-3} \\
\qquad \prod_{a=0}^{\left(5 d_{0}-1\right) / 2} \frac{a \lambda_{\mu(1)}+\left(5 d_{0}-a\right) \lambda_{\sigma(\mu(1))}}{d_{0}} \\
(-1)^{\left(d_{0}-1\right) / 2} \frac{d_{0} !}{d_{0}^{d_{0}}}\left(\lambda_{\mu(1)}-\lambda_{\sigma(\mu(1))}\right)^{d_{0}} \prod_{\substack{\left(d_{0}-1\right) / 2 \\
k \neq \mu(1), \sigma(\mu(1))}}^{\left(\frac{a}{d_{0}} \lambda_{\mu(1)}+\frac{d_{0}-a}{d_{0}} \lambda_{\sigma(\mu(1))}-\lambda_{k}\right)}
\end{gathered}
$$

Here, it is understood that the torus weights satisfy $\lambda_{2}=-\lambda_{1}, \lambda_{4}=-\lambda_{3}, \lambda_{5}=0$. Note that setting $\lambda_{5}$ to zero introduces zero weight components in the above formula, which however always exactly cancel between numerator and denominator. In formula (4.7), it is also understood that in counting the valence of the vertex called 1 , the half edge counts full.

The final formula is

$$
\tilde{n}_{d}=2 \sum_{\Gamma, \text { labellings }} \frac{1}{|\operatorname{Aut} \Gamma|} \int_{\overline{\mathcal{M}}_{\Gamma}} \frac{\mathbf{e}\left(\mathcal{E}_{d}^{\mathbb{R}}\right)}{\mathbf{e}\left(N_{\mathcal{M}}^{\mathbb{R}}\right)}
$$

As in [31], $\mid$ Aut $\Gamma \mid$ is the product of the order of the automorphism group of $\Gamma$ as a decorated graph times the product of the degrees on the edges (including $d_{0}$ ). For the first few degrees, one reproduces the results from eq. (2.25) in section 2.

Acknowledgments My interest in this problem was revived when Jake Solomon told me that the number of degree 1 holomorphic disks was 30. I would like to thank him for several helpful discussions and for sharing parts of his thesis. I am indebted to Katrin Wehrheim for patiently explaining what could (and could not) be learned from 
FO $^{3}$. I would also like to thank Simeon Hellerman, Calin Lazaroiu, Wolfang Lerche, Andy Neitzke, Rahul Pandharipande, and Edward Witten for valuable discussions and Dan Freed and Frank Sottile for helpful correspondence. This work was supported in part by the DOE under grant number DE-FG02-90ER40542.

\section{References}

[1] P. Candelas, X. C. De La Ossa, P. S. Green and L. Parkes, "A Pair Of Calabi-Yau Manifolds As An Exactly Soluble Superconformal Theory,” Nucl. Phys. B 359, 21 (1991).

[2] K. Fukaya, Y.-G. Oh, H. Ohta and K. Ono, "Lagrangian intersection Floer theoryanomaly and obstruction", preprint (2000)

[3] M. Kontsevich, "Homological algebra of mirror symmetry," Proceedings of I.C.M., Vol. 1,2 (Zürich, 1994) 120-139, Birkhäuser, 1995. [arXiv:math.ag/9411018].

[4] M. Aganagic and C. Vafa, "Mirror symmetry, D-branes and counting holomorphic discs," arXiv:hep-th/0012041.

[5] M. Aganagic, A. Klemm and C. Vafa, "Disk instantons, mirror symmetry and the duality web," Z. Naturforsch. A 57, 1 (2002) [arXiv:hep-th/0105045].

[6] P. Mayr, "N = 1 mirror symmetry and open/closed string duality," Adv. Theor. Math. Phys. 5, 213 (2002) [arXiv:hep-th/0108229].

[7] W. Lerche, P. Mayr and N. Warner, "Holomorphic N = 1 special geometry of open-closed type II strings," arXiv:hep-th/0207259.

[8] W. Lerche, P. Mayr and N. Warner, " $\mathrm{N}=1$ special geometry, mixed Hodge variations and toric geometry," arXiv:hep-th/0208039.

[9] A. Polishchuk and E. Zaslow, "Categorical mirror symmetry: The Elliptic curve," Adv. Theor. Math. Phys. 2, 443 (1998) [arXiv:math.ag/9801119].

[10] I. Brunner, M. Herbst, W. Lerche and J. Walcher, "Matrix factorizations and mirror symmetry: The cubic curve," arXiv:hep-th/0408243.

[11] E. Witten, "Chern-Simons gauge theory as a string theory," Prog. Math. 133, 637 (1995) [arXiv:hep-th/9207094].

[12] I. Brunner, M. R. Douglas, A. E. Lawrence and C. Romelsberger, "D-branes on the quintic," JHEP 0008, 015 (2000) [arXiv:hep-th/9906200].

[13] A. Recknagel and V. Schomerus, "D-branes in Gepner models," Nucl. Phys. B 531, 185 (1998) [arXiv:hep-th/9712186].

[14] I. Brunner, K. Hori, K. Hosomichi and J. Walcher, "Orientifolds of Gepner models," arXiv:hep-th/0401137.

[15] K. Hori and J. Walcher, "F-term equations near Gepner points," HEP 0501, 008 (2005) [arXiv:hep-th/0404196]. 
[16] K. Hori and J. Walcher, "D-branes from matrix factorizations," Talk at Strings '04, June 28-July 2 2004, Paris. Comptes Rendus Physique 5, 1061 (2004) [arXiv:hep-th/0409204].

[17] A. Kapustin and Y. Li, "D-branes in Landau-Ginzburg models and algebraic geometry," JHEP 0312, 005 (2003) [arXiv:hep-th/0210296].

[18] I. Brunner, M. Herbst, W. Lerche and B. Scheuner, "Landau-Ginzburg realization of open string TFT," arXiv:hep-th/0305133.

[19] K. Hori et al., "Mirror Symmetry," Clay Mathematics Monographs, Vol. 1, AMS 2003.

[20] C. Vafa, "Extending mirror conjecture to Calabi-Yau with bundles," arXiv:hep-th/9804131.

[21] H. Ooguri and C. Vafa, "Knot invariants and topological strings," Nucl. Phys. B 577, 419 (2000) [arXiv:hep-th/9912123].

[22] S. Kachru, S. Katz, A. E. Lawrence and J. McGreevy, "Open string instantons and superpotentials," Phys. Rev. D 62, 026001 (2000) [arXiv:hep-th/9912151].

[23] S. Govindarajan, T. Jayaraman and T. Sarkar, "Disc instantons in linear sigma models," Nucl. Phys. B 646, 498 (2002) [arXiv:hep-th/0108234].

[24] W. Lerche and P. Mayr, "On N = 1 mirror symmetry for open type II strings," arXiv:hep-th/0111113.

[25] S. Katz and C. C. Liu, "Enumerative Geometry of Stable Maps with Lagrangian Boundary Conditions and Multiple Covers of the Disc," Adv. Theor. Math. Phys. 5, 1 (2002) [arXiv:math.ag/0103074].

[26] T. Graber and E. Zaslow, "Open string Gromov-Witten invariants: Calculations and a mirror 'theorem'," arXiv:hep-th/0109075.

[27] P. Mayr, "Summing up open string instantons and $\mathrm{N}=1$ string amplitudes," arXiv:hep-th/0203237.

[28] C.-C. M. Liu, "Moduli space of J-Holomorphic Curves with Lagrangian Boundary Conditions and Open Gromov-Witten Invariants for an $S^{1}$-Equivariant Pair" arXiv:math.sg/0210257.

[29] J. Solomon, "Intersection Theory on the Moduli Space of Holomorphic Curves with Lagrangian Boundary Conditions in the Presence of an Anti-symplectic Involution," Introduction to Ph.D. Thesis, MIT (2006).

[30] J. Solomon, "Open Gromov-Witten theory," Talk at Columbia University, March 24, 2006 .

[31] M. Kontsevich, "Enumeration of rational curves via torus actions," arXiv:hep-th/9405035.

[32] J. Solomon, private communication.

[33] G. Ellingsrud and S. Strømme, "Bott's formula and enumerative geometry," J. AMS 9, 175-193 (1996) [arXiv:alg-geom/9411005]. 
[34] F. Sottile, "Enumerative Real Algebraic Geometry," in Algorithmic and Quantitative Aspects of Real Algbraic Geometry, S. Basu and L. Gonzalez-Vega, eds., DIMACS series 60, AMS, 2003. pp. 139-180; online at www.math.tamu.edu/ sottile

[35] J.-Y. Welschinger "Invariants of real symplectic 4-manifolds and lower bounds in real enumerative geometry" arXiv:math.ag/0303145.

[36] K. Hori, K. Hosomichi, D. C. Page, R. Rabadan and J. Walcher, "Non-perturbative orientifold transitions at the conifold," JHEP 0510, 026 (2005) [arXiv:hep-th/0506234].

[37] J. Walcher, unpublished.

[38] M. Bershadsky, S. Cecotti, H. Ooguri and C. Vafa, "Kodaira-Spencer theory of gravity and exact results for quantum string amplitudes," Commun. Math. Phys. 165, 311 (1994) [arXiv:hep-th/9309140].

[39] C. I. Lazaroiu, "String field theory and brane superpotentials," JHEP 0110, 018 (2001) [arXiv:hep-th/0107162].

[40] A. Tomasiello, "A-infinity structure and superpotentials," JHEP 0109, 030 (2001) [arXiv:hep-th/0107195].

[41] J. Walcher, "Stability of Landau-Ginzburg branes," J. Math. Phys. 46, 082305 (2005) [arXiv:hep-th/0412274].

[42] D. Orlov "Derived categories of coherent sheaves and triangulated categories of singularities" arXiv:math.ag/0503632.

[43] A. Kapustin and Y. Li, "Topological correlators in Landau-Ginzburg models with boundaries," arXiv:hep-th/0305136.

[44] M. Herbst and C. I. Lazaroiu, "Localization and traces in open-closed topological Landau-Ginzburg models," JHEP 0505, 044 (2005) [arXiv:hep-th/0404184].

[45] M. Herbst, W. Lerche and D. Nemeschansky, "Instanton geometry and quantum A(infinity) structure on the elliptic curve," arXiv:hep-th/0603085.

[46] M. Herbst, C. I. Lazaroiu and W. Lerche, "Superpotentials, $A_{\infty}$ relations and WDVV equations for open topological strings," arXiv:hep-th/0402110. 